\title{
CAPITULO 04
}

\section{A IMPORTÂNCIA DA EQUIPE MULTIDISCIPLINAR EM TEMPOS DA COVID-19: REVISÃO DE LITERATURA}

\section{DOI 10.4322/978-65-995353-2-1.c04}

\author{
Milena Kaory Kazume ${ }^{1}$, Mateus dos Santos Rios Matos ${ }^{2}$, Anderson Luís Paschoali ${ }^{3}$, \\ Abner Samuel dos Santos Lins ${ }^{4}$, Alice Freire dos Santos ${ }^{5}$, Luciana Canela de Siqueira \\ Silva ${ }^{6}$, Mariana Vieira Silva ${ }^{7}$, José Antonio Santos Souza ${ }^{8}$ \\ ${ }^{1}$ Universidade Brasil - Fernandópolis/SP, (milenakazume07@gmail.com) \\ ${ }^{2}$ Universidade Brasil - Fernandópolis/SP' (mateusrios1999@gmail.com) \\ ${ }^{3}$ Universidade Brasil - Fernandópolis/SP, (anderluispaschoali@gmail.com) \\ ${ }^{4}$ Universidade Brasil - Fernandópolis/SP, (abnerlins69@ gmail.com) \\ ${ }^{5}$ Universidade de Fortaleza - UNIFOR, Fortaleza, Ceará, (alicefreireds@edu.unifor.br) \\ ${ }^{6}$ Universidade Brasil - Fernandópolis/SP, (lucianacanela6@gmail.com) \\ ${ }^{7}$ Centro Universitário CESMAC - Maceió/AL, (maarivieira15@gmail.com) \\ ${ }^{8}$ Universidade Brasil - Fernandópolis/SP, (jose.ssouza@universidadebrasil.edu.br)
}

\section{Resumo}

Objetivo: Este trabalho tem como objetivo realizar uma revisão de literatura sobre a importância da equipe multidisciplinar em tempos da COVID-19, a fim de familiarizar os profissionais e estudantes da área da saúde. Método: Foi realizado um levantamento bibliográfico por meio de pesquisa nos bancos de dados SciELO, Biblioteca Virtual em Saúde, PubMed e Google Acadêmico, utilizando os termos descritores "Covid-19", "Equipe Interdisciplinar de Saúde" e "Atendimento Integral à Saúde". Resultados: As diversas pesquisas realizadas na área mostraram que a criação e atuação de equipes multidisciplinares no atendimento de pacientes com COVID-19 é fundamental para a plena recuperação das sequelas que podem vir a acometer estes pacientes. Conclusões: Diante da literatura revisada, pode-se concluir que é muito importante o trabalho em equipe de diferentes profissionais que estejam capacitados e que tenham o mesmo objetivo, o que trará eficiência na assistência à saúde.

Palavras-chave: Covid-19; Equipe Interdisciplinar de Saúde; Atendimento Integral à Saúde. Área Temática: Ciências da Saúde

E-mail do autor principal: $\underline{\text { milenakazume07@gmail.com }}$ 


\section{INTRODUÇÃO}

Em meados do mês de dezembro de 2019 foram registrados casos de uma nova infecção viral aguda grave em seres humanos na cidade de Wuhan, na China. A Organização Mundial da Saúde (OMS), em janeiro de 2020, declarou a infecção por COVID-19 (SARS-CoV-2) uma emergência de saúde mundial (O’BRIEN et al. 2020).

A pandemia se tornou um desafio de saúde pública atingindo vários países nos 5 continentes. No Brasil, foram registrados, até 7 de julho de 2020, 1.755 .779 casos e 69.184 mortes, causando assim, consequências sociais, econômicas, políticas, culturais, religiosas e de saúde, sejam físicas ou psicológicas (BELARMINO et al., 2020).

O novo Coronavírus possui uma alta taxa de transmissibilidade e provoca síndromes respiratórias agudas que podem variar de casos leves à casos graves; os leves correspondem a aproximadamente $80 \%$ dos casos de contaminação e, os graves aproximadamente 5 a $10 \%$ dos casos, sendo que estes últimos são associados à insuficiência respiratória e sua letalidade varia de acordo com as condições sistêmicas de cada indivíduo e faixa etária (GUIMARÃES et al., 2020).

Frente a essa problemática, Moradian (2020) cita uma estrutura de três camadas para colaboração entre diferentes disciplinas. A interdisciplinaridade é uma das 3 camadas citadas, propondo o trabalho conjunto entre profissionais de diferentes áreas de atuação a partir de uma base disciplinar específica, para enfrentar um problema comum.

A atuação de diferentes profisssionais no tratamento e reabilitação de pacientes que foram contaminados pelo Coronavírus, vai desde o diagnóstico até a reabilitação completa. Os profissionais da Enfermagem, Farmácia e Medicina fazem parte desta equipe; os profissionais da Fisioterapia e da Educação Física também podem fazer parte destas equipes atuando na reabilitação pulmonar durante e após a contaminação pelo vírus (KAPPEL; SILVA, RECKZIEGEL, 2020).

Nesse contexto, o compartilhamento de informações e conhecimentos entre os diferentes profissionais atuantes no combate à pandemia é de grande importância para a integridade nos cuidados de saúde, tornando-se crucial no combate à COVID-19 (SILVA et al., 2020).

Este trabalho tem como objetivo realizar uma revisão narrativa sobre a importância da equipe multidisciplinar em tempos da COVID-19, a fim de familiarizar os profissionais e estudantes da área da saúde. 


\section{MÉTODO}

O presente trabalho constituiu-se de uma revisão de literatura, de natureza descritivoqualitativa. A pesquisa bibliográfica foi realizada nas seguintes Bases de Dados: Scientific Eletronic Library Online (SciELO), Biblioteca Virtual em Saúde (BVS), PubMed e Google Acadêmico. Empregou-se os seguintes descritores: "COVID-19”, "Equipe Interdisciplinar de Saúde" e "Atendimento Integral à Saúde". O sistema de formulário avançado "AND” para filtragem dos artigos relacionados ao tema foi utilizado.

Os critérios de inclusão foram:

a) Possuir como temática central a equipe multidisciplinar e a COVID-19;

b) Artigos publicados no período de 2020 a 2021;

c) Artigos escritos em inglês, espanhol ou português.

Enquanto, os critérios de exclusão foram:

a) Teses e Dissertações.

b) Trabalhos que não compreendiam o tema da saúde.

\section{RESULTADOS E DISCUSSÃO}

A partir de um levantamento na Biblioteca Virtual em Saúde, ao utilizar os descritores citados anteriormente e submeter ao filtro, 2 artigos foram escolhidos para compor esta revisão. Já na plataforma da PubMed, utilizando os mesmos descritores, que passaram pelo filtro, 2 artigos foram selecionados. Na SciELO, utilizando os mesmos descritores e o filtro, para a confecção deste estudo, apenas 1 (um) foi selecionado, considerando a relevância do tema. No Google Acadêmico, 3 artigos foram escolhidos. Portanto, a partir dos critérios de inclusão e exclusão norteados pelo filtro, foram encontrados 54 artigos nas Bases de Dados. Após passar por uma análise e leitura na íntegra foram selecionados 8 artigos científicos que abordam sobre a importância da equipe multidisciplinar em tempos da COVID-19. A descrição dos artigos selecionados para análise encontra-se na Tabela 1.

\section{E - book Tripé do Ensino Superior: Ensino,} Pesquisa e Extensão 
Tabela 1: Autor/Ano/País, tipo de estudo, objetivo e achados dos artigos selecionados para a presente Revisão de Literatura.

\begin{tabular}{|c|c|c|c|}
\hline AUTOR/ ANO/ PAÍS & TIPO DE ESTUDO & OBJETIVO & ACHADOS \\
\hline $\begin{array}{l}\text { Belarmino et al. } 2020, \\
\text { Brasil }\end{array}$ & & $\begin{array}{l}\text { Relatar a vivência de práticas colaborativas } \\
\text { no enfrentamento da pandemia de COVID- } \\
19 .\end{array}$ & $\begin{array}{l}\text { As práticas colaborativas em equipe foram relevantes para organizar o fluxo de } \\
\text { trabalho diante do estresse nas emergências, do medo de contágio e conflitos. }\end{array}$ \\
\hline $\begin{array}{l}\text { Diaz, et al. 2021, } \\
\text { Norway }\end{array}$ & Relato de Caso & $\begin{array}{l}\text { Usar a situação Norueguesa como um caso } \\
\text { para explicar a necessidade de uma } \\
\text { abordagem interdisciplinar inovadora em } \\
\text { nível de sistema global. }\end{array}$ & $\begin{array}{l}\text { A COVID-19 deve ser confrontada por uma abordagem colaborativa } \\
\text { interdisciplinar, pois novas perspectivas que abordem como as múltiplas } \\
\text { dimensões estruturais da discriminação sistêmica em várias áreas da vida se } \\
\text { cruzam para impactar os resultados de saúde são necessárias. }\end{array}$ \\
\hline $\begin{array}{l}\text { Guimarães et al. 2020, } \\
\text { Brasil }\end{array}$ & Estudo Teórico Reflexivo & $\begin{array}{l}\text { Apresentar uma reflexão teórica acerca da } \\
\text { abordagem da equipe multiprofissional em } \\
\text { Nefrologia no cenário da COVID-19. }\end{array}$ & $\begin{array}{l}\text { A abordagem multiprofissional possibilita pensar em ações estratégicas } \\
\text { conjuntas por diversos profissionais que têm um objetivo em comum, } \\
\text { compreender o paciente com suspeita ou confirmação de COVID-19 e vincular } \\
\text { as ações a todas as esferas de direitos, o que permite alcançar melhores respostas } \\
\text { às suas demandas em sua totalidade, e não na perspectiva de fragmentação. }\end{array}$ \\
\hline Kappel et al. 2021, Brasil & Relato de Caso & $\begin{array}{l}\text { Analisar o cuidado multidisciplinar ao } \\
\text { paciente diagnosticado com COVID-19, do } \\
\text { diagnóstico à reabilitação pulmonar. }\end{array}$ & $\begin{array}{l}\text { O cuidado ao paciente com COVID-19, do diagnóstico à reabilitação pulmonar, } \\
\text { perpassa por diferentes áreas. Durante o período de internação hospitalar, } \\
\text { profissionais de Enfermagem, Farmácia, Medicina e Fisioterapia tiveram } \\
\text { atuação de destaque, garantido a estabilização do quadro de saúde do paciente. }\end{array}$ \\
\hline Moradian et al. 2020, Irã & Relato de Experiência & $\begin{array}{l}\text { Discutir a importância de reunir cientistas } \\
\text { de todo o mundo para encontrar soluções } \\
\text { eficazes para controlar a pandemia. }\end{array}$ & $\begin{array}{l}\text { O papel e a colaboração de Organizações Internacionais relacionadas à Saúde, } \\
\text { como a OMS, Organização para Alimentos e Agricultura (FAO), União } \\
\text { Internacional de Ciência da Nutrição (IUNS) e Organizações Não } \\
\text { Governamentais (ONGs), bem como consórcios internacionais, como Educação } \\
\text { Científica Universal e A Rede de Pesquisa (USERN) e academias nacionais e } \\
\text { internacionais, têm sido reconhecidas como cruciais para um conhecimento } \\
\text { integrado do novo Coronavírus, impactando no manejo efetivo da COVID-19 } \\
\text { em todo o planeta. }\end{array}$ \\
\hline
\end{tabular}


O’Brien et al. 2020, Irlanda Revisão de Literatura

Romeyke et al. 2020, Relato de Caso

Áustria

Relato de Experiência
Descrever a implantação do Serviço de Recuperação da COVID-19, serviço multidisciplinar para acompanhamento integral de pacientes com diagnóstico hospitalar de pneumonia por COVID-19.

Relatar um caso de um paciente infectado com síndrome respiratória aguda grave em decorrência da SARS-CoV-2.

Descrever as experiências de residentes multiprofissionais na Estratégia Saúde da Família acerca da aplicação de ações interdisciplinares no enfrentamento do novo Coronavírus.
Os autores demonstraram a implementação de uma abordagem multidisciplinar integrada aos pacientes com COVID-19, identificando aqueles com maiores necessidades de saúde física e mental. A experiência inicial dos autores sugere que deficiências físicas, psicológicas e cognitivas significativas podem persistir, apesar da resolução clínica da infecção.

A aplicação dos relatos dos pacientes pode ser útil para obter uma imagem mais abrangente de um paciente com COVID-19, em que "o paciente recebe uma voz", além de ser avaliado por outros. O conhecimento adquirido pode então ser disponibilizado para a equipe interdisciplinar de tratamento para ser incorporado ao plano de tratamento.

As práticas interdisciplinares têm se destacado nos mais diversos serviços de saúde como método de ascensão essencial para gestores, profissionais de saúde e estudantes na produção de relações mais atuantes de forma integrada no campo da saúde como um todo, principalmente no enfrentamento da pandemia. 
A partir da análise detalhada dos artigos selecionados fica evidente a importância do uso de uma equipe multidisciplinar no atendimento na área da saúde. Isso se tornou ainda mais evidente no período da pandemia da COVID-19, já que a doença é conhecida por deixar sequelas que podem levar a um longo período de tratamento e recuperação, denotando a necessidade da equipe multidisciplinar. Um exemplo dessa necessidade é a atual situação dos migrantes na Noruega; em todo o mundo, migrantes geralmente se enquadram em grupos mais vulneráveis, devido à grande parte advir de área de extrema pobreza ou fugindo de conflitos. Devido a essa vulnerabilidade, essas pessoas tem uma maior chance de adquirir doenças, especialmente as contagiosas como a COVID-19, e maior dificuldade no acesso aos tratamentos. Portanto, se torna indispensável o uso de uma equipe multidisciplinar no acompanhamento desses grupos (DIAZ et al., 2021).

Os pacientes com múltiplas comorbidades representam um outro grupo muito vulnerável à COVID-19, uma vez que esses pacientes possuem inúmeros problemas de saúde que podem ser exacerbados pela infecção respiratória. Romeyke et al. (2020) relataram um caso clínico de um paciente, sexo masculino, 84 anos de idade, que apresentava múltiplas comorbidades, dentre elas, pode-se destacar: diabetes mellitus tipo 2, hiperuricemia sintomática, estenose de aorta progressiva, falência renal crônica e hipertensão pulmonar. Seu quadro clínico se agravou pela COVID-19. Diante desse quadro, mostrou-se indispensável o uso de cuidado intensivo supervisionado por uma equipe multidisciplinar para estabilização e recuperação adequada do paciente para sua recuperação e controle das doenças associadas.

A despeito da importância da implantação de equipes multidisciplinares, poucos locais no mundo se dedicaram a essa árdua tarefa. Um ótimo exemplo da adaptabilidade do sistema público de saúde à nova situação foi a Irlanda. $\mathrm{O}$ país foi modelo no estabelecimento de um modelo híbrido, composto por clínicas virtuais e presenciais que se complementam no atendimento e prestação dos serviços de saúde. Essas equipes são compostas por serviços respiratórios, de cuidados intensivos, doenças infecciosas, psiquiatria e psicologia. Desse modo, a equipe multidisciplinar é capaz de identificar rapidamente as sequelas geradas pela COVID19 no paciente e prover a terapêutica adequada a cada caso, maximizando a recuperação plena dos acometidos (O’BRIEN et al., 2020).

Por fim, existem ótimos modelos e experiências de atuação de equipes multidisciplinares no Sistema Único de Saúde (SUS) e seus excelentes resultados para os pacientes atendidos. Recentemente, na capital do Ceará foi documentada a experiência de uso de uma equipe multidisciplinar com práticas colaborativas para atendimento de pacientes com 
COVID-19. A equipe é composta por médicos, enfermeiros e técnicos de enfermagem que trabalhavam de forma colaborativa usando o enfoque na interprofissionalidade e na interdisciplinaridade, a fim de prover uma melhor assistência à saúde e fortalecer a atuação da equipe. Apesar das dificuldades de implantação, o sistema se mostrou eficiente na melhora da qualidade do atendimento, na limitação de atrasos e erros e no controle do estresse da equipe no contexto dos atendimentos de COVID-19, devido ao medo de contágio e conflitos (BELARMINO et al., 2020).

Mediante a análise da literatura, foi possível constatar vários conceitos relativos à importância da cooperação interprofissional no manejo da COVID-19. De fato, de acordo com Silva et al. (2020), a cooperação entre os profissionais é um importante fator modificador das ações de cuidado efetuadas, pois baseia-se na resolução de problemas, potencialização da eficácia e qualidade nos cuidados, por meio da compreensão dos papéis e de competências colaborativas para o trabalho em equipe. Nesse sentido, Diaz (2021) ressalta que a cooperação interprofissional entre categorias surge como resposta à realidade da saúde mundial que exige novas formas de assistência, capazes de lidar com a multiplicidade dos indivíduos portadores, através da atuação de enfermeiros com habilidade em práticas avançadas, médicos, assistentes sociais, odontólogos, nutricionistas, fisioterapeutas e farmacêuticos.

Ademais, a fim de que a efetivação das mudanças na configuração assistencial ocorra, no contexto da COVID-19, Silva et al. (2020) verificaram que podem ser realizadas capacitações simuladas, envolvendo a equipe de assistência à saúde, acerca da colocação adequada de equipamentos de proteção individual, intubação orotraqueal e ventilação mecânica, assim como atuação diante de paradas cardiorrespiratórias em pacientes suspeitos.

Dentre as vantagens da prática interprofissional destacadas por Silva et al. (2020), ressalta-se a melhoria da experiência do paciente e da família no cuidado à saúde, bem como vantagens na relação custo-efetividade da atenção à saúde. Devido a isso, habilidades de atuação em equipe também devem ser empregadas durante a pandemia da COVID-19, como comunicação, respeito, confiança, conhecimento mútuo, poder, centralização das ações no paciente, características das tarefas e do ambiente.

Segundo Guimarães et al. (2020), os artigos relacionados a cuidados de suporte na COVID-19 recomendam que o estado nutricional deve ser avaliado em todos os pacientes infectados na admissão hospitalar e os pacientes em risco nutricional devem receber apoio nutricional o mais breve possível. Por esta razão, a atuação do nutricionista é indispensável. 
Já de acordo com Belarmino (2020), a equipe odontológica mostrou-se atuante em atividades generalistas e sanitárias, compondo as equipes de acolhimento, responsáveis pela organização do fluxo e encaminhamento de casos suspeitos para triagem, além de desenvolvimento de material audiovisual sobre a área para ser disponibilizado em redes sociais.

Outrossim, a Enfermagem se destaca na detecção e na avaliação dos casos suspeitos de COVID-19, devido à sua capacidade técnica e por estar mais tempo no acompanhamento do paciente. O profissional do Serviço Social tem a função de mediar, junto à equipe de saúde, a realidade social dos pacientes, buscando atuar nas demandas apresentadas pelo paciente em sua totalidade.

\section{CONCLUSÃO}

Diante do presente trabalho, pode-se concluir que, atualmente, a realidade da saúde mundial exige novas formas de assistência, e a equipe multidisciplinar é capaz de cooperar para um tratamento mais eficaz durante a doença e identificar rapidamente as sequelas geradas pela COVID-19 no paciente, fornecendo, assim, uma terapêutica adequada a cada caso.

É importante que os profissionais que atuam em equipes multidisciplinares estejam capacitados e que tenham o mesmo objetivo; isso trará eficiência na assistência à saúde, no controle e no combate à COVID-19, seguindo as recomendações estabelecidas pelas autoridades sanitárias competentes. 


\section{REFERÊNCIAS}

BELARMINO, A. C. et al. Práticas colaborativas em equipe de saúde diante da pandemia de COVID-19. Rev Bras Enferm. v. 73, p. 1-5, e20200470, ago. 2020. DOI 10.1590/00347167-2020-0470.

DIAZ, E. et al. Aprendendo com a pandemia COVID-19 entre os migrantes: é necessária uma abordagem inovadora e interdisciplinar em nível de sistema para melhorar a saúde pública. Scand Journal Pub Health. p. 1-5, maio 2021. DOI 10.1177/14034948211019795.

GUIMARÃES, A. S. M. et al. Atuação da equipe multiprofissional em saúde, no cenário da pandemia por Covid 19. Health Resid Journal. v.1, n.2, p. 1-22, maio 2020. DOI 10.51723/hrj.v1i2.37.

KAPPEL, R. H.; SILVA, P. T.; RECKZIEGEL, M. B. Atuação de equipe multidisciplinar no tratamento de um caso de infecção por coronavírus: relato de caso. Anais do Seminário Científico do Programa de Pós-Graduação em Promoção da Saúde da UNISC, Santa Cruz do Sul, 2020. Disponível em: https://online.unisc.br/acadnet/anais/index.php/scps/index. Acesso: 09 de jul. 2021.

MORADIAN, N. et al. A necessidade urgente de ciência integrada para combater a pandemia e além. Journal Transl Med. v. 18, n. 205, p. 1-7, maio 2020. DOI 10.1186/s12967-02002364-2.

O'BRIEN, H. et al. Um modelo multidisciplinar integrado de cuidados de recuperação COVID-19. Ir Journal Med Sci, Irlanda. v. 190, n. 2, p. 461-468, set. 2020. DOI 10.1007/s11845-020-02354-9.

ROMEYKE, T.; NOEHAMMER, E.; STUMMER, H. Relato de caso COVID-19: um homem de 84 anos com exacerbação de múltiplas comorbidades devido ao COVID-19 gerenciado por uma equipe multidisciplinar usando resultados relatados pelo paciente. Am Journal Case Rep. v. 21, p. 1-7, e926694, ago. 2020. DOI 10.12659.

\section{E - book Tripé do Ensino Superior: Ensino, Pesquisa e Extensão}


SILVA, R. S. et al. Práticas interdisciplinares no enfrentamento da COVID-19 na estratégia saúde da família. Enferm Foco, Brasília. v. 11, n. 2, p. 246-253, dez 2020. DOI 10.21675/2357-707X.2020.v11.n2.ESP.4220. 